\title{
Discover, analysis, and interpretation of problems and comparing satisfaction of hemodialysis and peritoneal dialysis patients: patients' perspective
}

Ghazanfar Rafiee ( $\nabla$ raf_gh2003@yahoo.com )

Shiraz University of Medical Sciences

Jamshid Roozbeh

Shiraz University of Medical Sciences

\section{Research}

Keywords: Hemodialysis, peritoneal dialysis, satisfaction, problems

Posted Date: February 28th, 2020

DOl: https://doi.org/10.21203/rs.2.24830/v1

License: (c) (i) This work is licensed under a Creative Commons Attribution 4.0 International License.

Read Full License 


\section{Abstract}

Background: End-stage renal disease is an irreversible and progressive loss of kidney function and it can be fatal without hemodialysis, peritoneal dialysis or kidney transplantation. Hemodialysis is a type of treatment where the patient is connected to a machine through a catheter via veins for twice or three times a week for approximately four hours. Alternatively, peritoneal dialysis is carried out with a plastic catheter insertion into the abdomen through which dialysis fluid (glucose) enters and is taken out.

This study aimed to discover, analyze, interpret and compare end-stage renal disease patient's satisfaction with hemodialysis and peritoneal dialysis with an emphasis on complications and problems that was created during dialysis treatment.

Methods: this study a qualitative exploratory approach was used at Shiraz hemodialysis and peritoneal dialysis centers 2017-2018. In these centers there were 345 ESRD patients who were receiving dialysis. A purposeful sample of 35 hemodialysis and 30 peritoneal dialysis patients were interviewed. The data were collected through interview. Each session lasted for 50 minutes. Initially 12 open-ended questions were developed and used to stimulate discussions in sessions. Directed content analysis was used for analyzing the transcribed data. After giving a code to each line or incidence, codes were then compared for similarity and differences, merged together, and categorized.

Results: Themes of Fatigue experience, Insomnia, Wasting time, Travel and leisure time activities limitations, Hypotension, Dissatisfaction and satisfaction with hemodialysis, Peritoneal catheter problems, peritoneal dialysis difficulties and limitations, satisfaction from peritoneal dialysis emerged. Each dialysis method has its own problems. Some problems and limitations were more emphasized on by patients.

Conclusion: Each dialysis method has its own problems. Some problems and limitations were more emphasized on by patients.

\section{Background}

In End-stage renal disease(ESRD) the body is incapable to maintain metabolic and electrolyte balance that leads to uremia, metabolic acidosis, anemia, and the endocrine system disorders. This disease can be fatal without hemodialysis, peritoneal dialysis or kidney transplantation[1, 2]. During Hemodialysis the patient is connected to a machine through a catheter via veins for twice or three times a week for approximately four hours[3,4]. $60 \%$ of hemodialysis patients treated in five countries (United State, Japan, Germany, Brazil, and Italy), but their population form only $12 \%$ of the world[5]. As a result of longterm continues hemodialysis treatment sessions (3-4 hour sessions, three times a week), dietary restrictions and medication regimen, patient has to go through major changes in their lifestyle. ${ }^{6}$ Alternatively, peritoneal dialysis is carried out with a plastic catheter insertion into the abdomen through which dialysis fluid enters and is taken out[1, 3].Continuous Ambulatory Peritoneal Dialysis (CAPD) technique is the most effective type of home care treatment and it allows the patient to be more 
independent and active[3]. CAPD technique is the most common type in Canada, the UK(United kingdom), Hong Kong and Mexico[7]. One of the advantages of this procedure is that patient's vein stays intact[8]. Infection is a major cause of failure and a drawback in peritoneal dialysis. Environmental and skin microorganisms contamination associated with manipulation of the transfusion set are the main causes of infection. During the past few decades, with improving and advancement in peritoneal catheter insertion, caring after its insertion, the importance of improvement in connector technology as well as the content of dialysis fluid, the result of peritoneal dialysis has significantly improved[9]. Some studies have shown a better quality of life in peritoneal patients in comparison with hemodialysis patients while other studies have shown similar physical quality for both, nonetheless they found higher mental quality in peritoneal dialysis patients[10]. This study aimed to discover, analyze, interpret and compare end-stage renal disease patient's satisfaction with hemodialysis and peritoneal dialysis with an emphasis on complications and problems that was created during dialysis treatment.

\section{Methods}

In this study a qualitative exploratory approach was used at Shiraz hemodialysis and peritoneal dialysis centers 2017-2018. In these centers there were 345 ESRD patients who were receiving dialysis (177) hemodialysis and (168) peritoneal dialysis.

\section{Study design and participants}

A purposeful sample of 35 hemodialysis and 30 peritoneal dialysis patients were interviewed and the data on their opinions about hemodialysis and peritoneal dialysis method, their physical and psychological problems, complications and satisfaction were collected through face-to-face deep semistructured interviews. Each interview session lasted for at least 50 minutes. Data collection and analysis proceeded concurrently and once the themes were identified and data saturation was achieved, the interviews were discontinued. Prior to recording the interviews, the objective of the study was verbally clarified for each participant and the participants' concerns were answered. Initially 12 open-ended questions that were related to their dialysis status, physical, psychosocial problems before, during, and after dialysis with participants' satisfaction from dialysis modalities were developed and used to stimulate discussions in the interview sessions.

\section{Data Analysis}

Directed content analysis was used in order to analyze the transcribed data. Initial analysis focused on understanding the information and developing codes and categories through identifying the emphasized words, phrases, themes, or concepts within the data[11]. The analytic process was utilized to become familiar with the data during the transcription and translation. For coding the transcript, it was necessary to go through the transcripts line by line and paragraph by paragraph to look for a significant statement and code based on the topics which they had addressed. Overall, three levels of coding were selected as appropriate. 


\section{Level 1}

Examining the data line by line and extracting codes taken from the interviews with subjects who attended the interviews.

\section{Level 2}

Comparing the coded data with each other to create categories. In fact, the categories were simply the coded data which seemed to cluster that may have resulted from condensing the codes.

\section{Level 3}

Coding is the title given to the central themes that emerged from the categories[12]. Initially the recorded interviews were transcribed, read, and re-read on a number of occasions to get an overall feeling of what the participants were saying. Each line or incident described was then given a code, which reflected the essence of the participants' comments. Codes were then compared for similarity and differences, merged together, and categorized. Table 1 shows three levels of codes for one of the themes. The measures used in the present study was to establish the rigor including; "truth value," "applicability," "consistency," and "neutrality" [13].

Table 1

Examples of coding

\begin{tabular}{|lll|}
\hline Level 1 codes (meaning unit) & $\begin{array}{l}\text { Level } 2 \text { codes } \\
\text { (categories) }\end{array}$ & $\begin{array}{l}\text { Level } 3 \text { codes } \\
\text { (theme) }\end{array}$ \\
\hline $\begin{array}{l}\text { Diminished strength and getting sleepy after } \\
\text { dialysis }\end{array}$ & Physical limitations & Wasting time \\
\hline The delay in the onset of dialysis & & \\
\hline $\begin{array}{l}\text { Visiting dialysis center three times a week } \\
\begin{array}{l}\text { Spending at least } 4 \text { hours for each dialysis } \\
\text { session }\end{array}\end{array}$ & lack of time & \\
\hline
\end{tabular}

\section{Results}

The qualitative content analysis of data led to the emergence of nine major themes from the deep interview data. From hemodialysis patients' points of view six themes and peritoneal dialysis patients' points of view, three themes were considered as the important themes:

Patients' problems from Hemodialysis

A: Experiencing fatigue

Diminished ability: 
This theme showed that hemodialysis participants spoke of sluggishness and inability that occurred after dialysis. They stated that they felt tired after each hemodialysis session and when they were walking they felt fatigue and didn't have the ability to work.

One of the participants stated that

When I come home due to fatigue I need to rest. When I walk 200-300 meters, I feel tired. I can't work, quickly I get tired, I can't lift anything.

B: Insomnia

Sleep disturbance

Some hemodialysis participants had trouble sleeping. They complained of having problem in falling asleep and staying asleep. Frequent awakening from night sleep and wakening up early in the morning was another sleep disturbance in these patients.

One of the participants mentioned that

I can't go to sleep until 12 o'clock midnight and then I wake up after 2-3 hours and I'm not able to go back to sleep again.

Another one said

I'm restless during night sleep. The days that I go for dialysis, I only sleep between 1-1.5 hr at night. Then I don't sleep until morning and I get frustrated, but the night after it gets better.

C: Wasting time

\section{Lack of time}

Hemodialysis patients usually have to be present for receiving treatment in dialysis ward at a certain time. All of these mostly depend on patient's home distance from the hemodialysis center which may take a while. Even though when patients go to dialysis center based on predetermined appointments, but some unforeseen events may influence these delays such as; number of beds and number of patients admitted. These delays may cause distress in a person who has tried to reach the center on time. Also performing a physical examination and taking information before each dialysis session, is time consuming. After starting dialysis, if there are no difficulties during the session they have to stay connected to dialysis machine for a long period of time.

One of the participants said that

I have been standing here for 5 hours and this is the main reason why I feel sick. I had to wait for 5 hours. Half of my life is wasted. When I come here my time is wasted, I come here every other day.

One other participant stated that

during the days that I come for the dialysis, I can't do anything or meet up with my family and friends, I just sleep and relax.

D: Physical limitations

The studied hemodialysis participants were disappointed due to reducing their physical abilities. Owing to fatigue and physical limitations associated with dialysis, patients were bored and sleepy and their useful part of day was wasted. Because of their poor physical condition and spending most of their valuable time sleeping, they cannot participate in their favorite activities. 
One participant stated

After dialysis I cannot do anything for 10-12 h and up to $24 \mathrm{~h}$ after dialysis my body is exhausted, I'm sluggish, have nausea and headache and I sleep all the time. When I leave this place, I straight go to bed. After dialysis, my day is completely ruined, when I go home I feel bored and sleepy, it seems that I have fallen down from a mountain and I am incapable of doing anything.

E: Travel and leisure time activities limitations

Not having a suitable condition for traveling

Seemingly, due to continuity of hemodialysis, hemodialysis patients are less concern about traveling. Even if they think about traveling because of fatigue and loss of power, it becomes impossible for them to travel. Since it's necessary for hemodialysis patients to anticipate and coordinate everything concerning the place that they intend to travel to, have to have dialysis facilities, this makes it too hard for them to travel.

One participant stated

I traveled once, but the conditions for going to a party and travel was not possible because hemodialysis facilities were not available everywhere. Even if facilities were available in the place where I traveled to, but finding and coordinating with the nearest center was a very difficult thing to do. When I want to go on a trip, first I forget I am on dialysis, and when I do realize my condition I feel it's better to stay at home.

F: Hypotension

Dizziness, sluggishness and weakness

Our hemodialysis participants also suffered from dizziness, sluggishness and weakness after each session. They expressed their concern by saying that they felt dizzy when they went for a walk. According to patient's statements, this feeling occurred following dialysis due to reduction in blood pressure.

One of the participants stated that sometimes, I can't go out the day after dialysis, my blood pressure is slightly low, the next day when I get up I feel like I'm going to fall. Sometimes, after dialysis I am dizzy. When I am connected to the machine my blood pressure falls. Because of this, they administer intravenous fluid and I get better. Sometimes after dialysis I feel I'm going to fall in the middle of a street, I do not have the strength to walk on my legs, then I immediately sit down.

G: Dissatisfaction and Satisfaction from hemodialysis

Although half of hemodialysis participants described the above mentioned problems, the other half of the participants who underwent dialysis three times a week stated that they did not have any of these problems and were satisfied with their dialysis before, during and after each session. They had adapted themselves with their condition.

One of the hemodialysis participants who were dissatisfied whit his/her condition stated that After dialysis I'm not able to work and I feel exhausted. I am dissatisfied with the duration which is time consuming. Diet regimen has created restriction for me. I like to drink soft drinks, but I can't. It has affected my socializing; I don't participate in wedding or funeral ceremonies. My sex life has gone down the drain. I easily get stressed-out and bored. 
And one the hemodialysis participant who was satisfied with his/her dialysis stated

I don't have any problem before, during or after dialysis. I get a good night sleep. I don't have anxiety. I do my daily routine activities. I travel abroad. I don't have any problem. I am satisfied with my hemodialysis. This is better in comparison with other methods.

Patients' problems and concerns with peritoneal dialysis

A: Peritoneal Catheter problems

Physical problems related to peritoneal catheter

One of the peritoneal dialysis participant's problems was a sense of fluid volume in the abdomen and pain at the end of fluid drainage from the peritoneal space. They felt these problems during the first few sessions but then these feelings disappeared over time.

One of the participants stated that when I perform dialysis, at the end of each session due to withdrawing mechanism, I feel pain. I feel pressure in my abdomen when there is fluid, it squeezes my chest.

Mechanical problems related to peritoneal catheter

Despite the advances in peritoneal dialysis technology, the risk of infection is still considered as one of the major concerns for these patients.

One of the participants in this case pointed out that

I have changed the catheter twice because it was infected. Tunnel was infected, I had to use medication, but my condition hasn't improved. Catheter Cuff had come out.

B: Peritoneal dialysis difficulties and limitations

For performing peritoneal dialysis, patient has to be comfortable, designate a place, anticipate and plan time for executing it. It might be difficult finding a suitable place to do this for those who are not at home. Especially those who are employed or planning to travel are faced with many restrictions. For those who want to travel, they need to coordinate, transfer solution and find a source to obtain the solution at the destination. Providing and planning of solution is a concern for peritoneal dialysis patients, and one of the participants stated that: Performing peritoneal dialysis has time and place limitation. If the solution volume and number of fluid exchanges were less frequent it was better. For example, if it is done twice a day, it is better. I have restriction for lifting objects I can't lift anything heavier than $2 \mathrm{~kg}$. I feel it is difficult to go on a vacation. At first, I thought I could go and see my daughter who lives abroad but it is hard for me. I was going to stay for a month, how was I going to do my dialysis?

C: Satisfaction from peritoneal dialysis

At first, performing peritoneal dialysis appeared to be a bit difficult for the participants in the study and minor problems were encountered, but gradually they got used to the technique, these problems were rectified and they were more satisfied with their dialysis. These participants did not suffer from fatigue, insomnia, daytime sleepiness, hypotension that most hemodialysis patients were suffering from.

One participant stated that

Now it has been a month since a catheter was inserted, when I go out I don't feel a thing and I don't notice it, I forget that the catheter is in my abdomen. I can socialize without any limitations. I don't have any diet 
restrictions. Before I started dialysis, I had hypertension, but since I have been performing peritoneal dialysis, my blood pressure is under control.

In this regard another participant pointed out I don't have restriction in my activities. Before going out I perform my dialysis. I don't have a drainage problem, but at the end I feel a little bit of pain. It is better than hemodialysis. You can take care of yourself. It doesn't waste my time.

\section{Discussion}

One of the most common problems that hemodialysis participants frequently complained of it was fatigue. Fatigue, is the most common and is the severest symptom in hemodialysis patients[14]. For many hemodialysis patients, on regular basis after each dialysis session felling of fatigue occurs[15].

Wasting time theme suggested waiting in the ward before the commencement of dialysis session, having to go there three times a week and each time for 4 hours in the dialysis ward and spending time to recover after dialysis. Although hemodialysis patients refer to centers based on appointments, but sometimes their session doesn't start on time, and the amount of time they must wait is too long. They had to wait before each dialysis session, and they have to wait after dialysis due to related changes that occur in their physical condition. These patients aren't satisfied since their time is being wasted and have to spend more than the usual time in an unsuitable condition. Our findings suggested that hemodialysis participants seem to be upset for losing their time because of treatment.

Although hemodialysis participants were willing to travel and thought of traveling, but they lost their motivation because of problems and loss of physical strength attributed to hemodialysis. Even if they had the motivation and ability to travel, they must coordinate with their destination hemodialysis center for continuing their treatment and at the destination facilities must be available to perform hemodialysis, and/or referred to a city or a location near their destination where it has dialysis facilities.

Despite the fact that the studied hemodialysis patients were sleepy and tired after dialysis and felt weak, however, they couldn't get enough sleep especially in the evening after dialysis session. These patients usually suffered from not being able to sleep until late at night, frequent awakening during the night and not being able to go back to sleep after waking up. It seems that taking a nap during hemodialysis makes more problems with night sleeping for them. Since peritoneal dialysis patients are active while the dialysis process, therefore, they sleep less during the day, so they have a better night sleep[16].

Some studied peritoneal dialysis patients suffered from fluid drainage pain in their abdomen at the end of each session. But gradually this feeling disappeared. Initially, peritoneal dialysis catheter was felt like a foreign object in the abdomen, but this feeling was gradually reduced over time.

Hygiene, cleanliness and preventing catheter contamination are critical in peritoneal dialysis. Since not everyone can comply with these instructions or due to space limitation, location and the proximity of peritoneal dialysis catheter to the digestive system, some people are more susceptible to catheter 
contamination. Since peritoneal catheter infection is the most common and serious complication of peritoneal dialysis, and despite the fact that participants had been trained to observe sterility, but some of the participants were concerned about catheter infection.

Although dialysate is sufficiently provided, but these patients have to prepare the equipment four times a day and each time for 30-40 minutes as well as exchanging the dialysate, hence, performing these steps are time consuming. Despite being time-consuming the spent time seems reasonable, but for some patients this producer seems too long. Since this type of dialysis should be performed in a suitable location which is not contaminated, but sustaining such an environment is not always possible, sometimes creating such an environment during a trip or work place is too difficult and according to them, this is a major limitation for this type of dialysis. Another limitation of this method as experienced by hemodialysis patients was going on a vacation. If the dialysate solution was not available in the destination, transferring high volume of this fluid was too hard and it was not feasible. Although unemployed and housewives did not need to carry dialysate and they could do dialysis at home, but it was more difficult for people who worked to take the fluid along with themselves as well as finding a suitable place at workplace and this was considered as another limitations in this approach. Since peritoneal dialysis patients are not dependent on the availability of dialysis ward, travelling is easier for them. Peritoneal dialysis patients need a pre-determined and arranged plan for medical care support arrangement during their journey. These people may need to contact peritoneal dialysis center where they wish to go on a vacation and request assistance if any problem arise. If they want to stay for a long period of time it is better that they send the required equipment to the destination before travelling and make sure that the equipment has reached the destination. Before travelling, these patients need to designate a clean space to conduct dialysis fluid exchange[17].

Mainly, the number and diversity of complications were much higher in hemodialysis patients. Since peritoneal dialysis patients experienced infection directly, from these patients' perspective, infection seemed to be a very big problem. If during fluid exchange the trained hygiene procedures were observed as well as with advancement in providing peritoneal dialysis equipment and surgery, peritoneal dialysis patients' problems were less than hemodialysis ones. There are many advantages in peritoneal dialysis such as; capability of executing it at home, maintaining residual renal function, fluid, blood pressure control, being cost effective, reducing the number of mortality and better quality of life[18].

Our data have shown that peritoneal dialysis patients are more satisfied with their treatment and they believed that their treatment had less impact on their life in comparison with hemodialysis patients. Peritoneal dialysis patients have significantly less co-morbidity in comparison with hemodialysis patients. Studies have shown that patients who use peritoneal dialysis feel more freedom and are able to manage their own treatment[19]. Patients who use peritoneal dialysis showed to be more flexible with regards to the time of dialysis[20].

\section{Conclusions}


Each dialysis method has its own problems and limitations. However, some patients have little problem and are satisfied with both dialysis method. Some problems and limitations were more emphasized on by patients. Hemodialysis problems and patients' dependency on hemodialysis machine and ward is more and patients' satisfaction were less. Peritoneal dialysis patients, who do not get infection, are satisfied with their peritoneal dialysis method. In peritoneal dialysis, patients' limitations and problems are less and they are relaxed and have more freedom. Due to peritoneal infection, peritoneal dialysis patients are more anxious. Some factors are dependent on the will of patients to prevent infection in peritoneal dialysis that must be observed, but some other parameters are uncontainable, but if patients become aware of them, they can reduce risk factors in order to experience better quality of life.

\section{Declarations}

\section{Abbreviations}

ESRD, CAPD, UK

\section{Acknowledgements}

The authors would like to thank all the people who helped to conduct this study. The authors wish to thank Dr. Nasrin Shokrpour and Mr. H. Argasi at the Research Consultation Center (RCC) of Shiraz University of Medical Sciences for his invaluable assistance in editing this manuscript.

\section{Authors'contributions}

This study was conceived, designed, and made substantial contributions to the acquisition of data by Gh $\mathrm{R}$, and JR made substantial contribution to the analysis and interpretation of data. Gh $\mathrm{R}$ wrote the first draft and JR critically reviewed the manuscript and provided important intellectual content. All authors approved the final manuscript for publication. Interviews were undertaken by GhR. Gh R, wrote the first draft. All authors reviewed all drafts and approved the final submitted manuscript.

\section{Funding}

This study has been supported by Shiraz NephroUrology Research Center. SNRC is supported by the Shiraz University of Medical Sciences.

\section{Availability of data and materials}

The datasets used and/or analysed during the current study are available from the corresponding author on reasonable request.

\section{Ethics approval and consent to participate}

This article does not contain any studies with animals performed by any authors. Informed consent was obtained from the patient 


\section{Consent for publication}

Not applicable.

\section{Competing interests}

The authors declare that they have no competing interests.

\section{Author details}

${ }^{1}$ Department of Medical and Surgical Nursing, Nursing and Midwifery School, Shiraz University of Medical Sciences, Shiraz, Iran. ${ }^{2}$ Shiraz NephroUrology research center, Shiraz University of Medical Sciences, Shiraz, Iran.

\section{References}

1. Baillie J, Lankshear A. patients' and relatives' experiences of peritonitis when using peritoneal dialysis. Journal of Renal Care. 2015; 41(3): p. 177-86.

2. Gerogianni SK, Babatsikou FP. Psychological Aspects in Chronic Renal Failure. Health science journal. 2014; 8(2): p. 205-214.

3. Kovac JA. The Relationships between Patient Satisfaction with Care and Depression and Compliance in End-Stage Renal Disease Patients Being Treated with Hemodialysis. (The George Washington University) [UMI proquest digital Dissertations]. 1997.

4. Keyser BL. Nephrology Nurses' Job Satisfaction in Chronic Hemodialysis Outpatient Facilities and the Relationship between Nephrology Nurses' Job Satisfaction and Intent to Stay. The George Wilmington University, UMI. 2011.

5. Bamgboye EL. Hemodialysis: Management problems in developing countries, with Nigeria as a surrogate. Kidney International. 2003; 83: p. S93-95.

6. Davis PG. Satisfaction with Health Care: A Predictor of Hemodialysis Patient Compliance. LSU Historical Dissertations and Theses. http://digitalcommon s.Isu.edu/gradschol_disstheses/6243. 1996.

7. Munib S. continuous ambulatory peritoneal dialysis (CAPD). Gomal Journal of Medical Sciences. 2006; 4(2): p. 82-85.

8. Baillie J, Lankshear A, Featherstoneet K. Perspectives on peritoneal dialysis at home: implications for the management of a chronic condition: A study protocol.Journal of advanced nursing $2011 ; 68(8)$ : p. 1847-1857.

9. Li PK, Szeto CC, Piraino B, et al. Peritoneal dialysis-related infections recommendations update. Perit Dial Int. 2010; 30(4): p. 393-423.

10. Liem YS, et al. Quality of Life Assessed with the Medical Outcomes Study Short Form 36-Item Health Survey of Patients on Renal Replacement Therapy: A Systematic Review and Meta-Analysis. Value 
Health. 2007; 10(5): p. 390-7.

11. Richards L, Morse JM. Read Me First for a User's guide to Qualitative Methods. 3rd edition, ed. 2013: Thousand Oaks, CA: Sage Publications.

12. Polit DF, Bernadette PH. Nursing research: Principles and Methods. 8th ed. Lippincott Williams and Wilkins. 2008.

13. Guba EG, Lincoln YS. Effective evaluation improving the Usefulness of Evaluation Results through Responsive and Naturalistic Approaches. San Francisco: Jossey-Bass 1992.

14. Jablonski A. The multidimensional characteristics of symptoms reported by patients on hemodialysis. Nephrology Nursing Journal. 2007; 34(1): p. 29-37.

15. Mollaoglu M. Fatigue in People Undergoing Hemodialysis. Dialysis \& Transplantation. 2009; 38(6): p. 216-20.

16. Eghbali M, Shahqolian N, Nazari F, at al. Comparing problems of patients with chronic renal failure undergoing hemodialysis and peritoneal dialysis referring to medical university's hospitals Iranian Journal of Nursing and Midwifery Research. 2009; 14(1): p. 1-5.

17. Corr C. A "New Normal": Life on Dialysis-The First 90 Days. Evailable at[https://www.kidney.org/sites/default/files/docs/11-100307_dialysistransitionbk2_oct07_Ir_bm.pdf

18. Asif A, Merrill D, Leon C, et al. Strategies to Minimize Tunneled Hemodialysis Catheter Use. Blood Purif. 2006; 24(1): p. 90-94.

19. Chanouzas D, Ng KP, Fallouh B, et al. What influences patient choice of treatment modality at the pre-dialysis stage. Nephrol Dial Transplant. 2012; 27(4): p. 1542-1547.

20. Baillie J. Perspectives on peritoneal dialysis at home: an ethnographic study: A thesis submitted in partial fulfilment of the requirements of Cardiff University for the degree of Doctor of Philosophy. A thesis submitted in partial fulfilment of the requirements of Cardiff University for the degree of Doctor of Philosophy. 2013. https://orca.cf.ac.uk/52540/1/2013bailliejphd.pdf. 